\title{
MRT-Bildgebung von Orbitaerkrankungen im Kindesalter
}

\section{Einleitung \\ $\nabla$}

Orbitaerkrankungen im Kindesalter sind selten und unterscheiden sich von denen im Erwachsenenalter. Trotz enger anatomischer Verhältnisse gibt es zudem eine

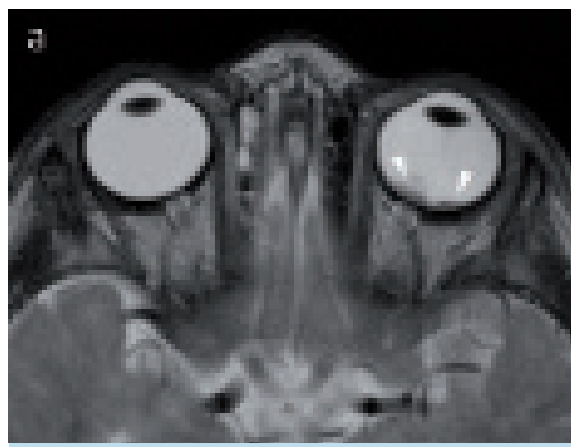

Vielzahl unterschiedlicher orbitaler Pathologien. Die meisten Orbitatumoren sind benigne, aber auch ohne Infiltration der Umgebung kann ebenso wie bei malignen Erkrankungen Visus und Leben des Kindes durch ein rasches Tumorwachs-

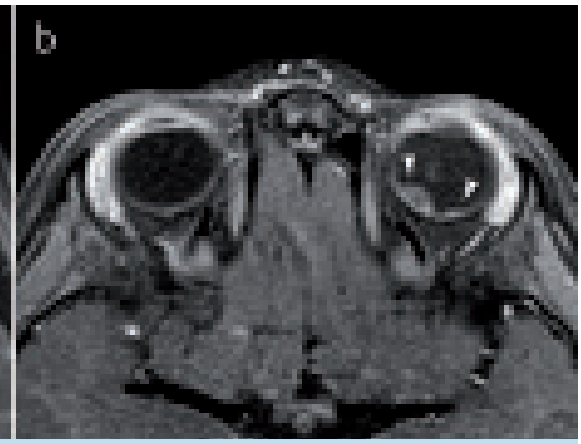

Abb. 1 Retinoblastom bei einem 2-jährigen Jungen. Das axiale T2-gewichtete Bild (a) und das fettgesättigte T1-gewichtete Bild nach Kontrastmittelgabe (KM = Kontrastmittel, b) zeigen eine diskrete Verdickung des dorsalen Bulbus mit einer gering verstärkten KM-Aufnahme. Ein intrakonales Wachstum ist nicht nachweisbar.
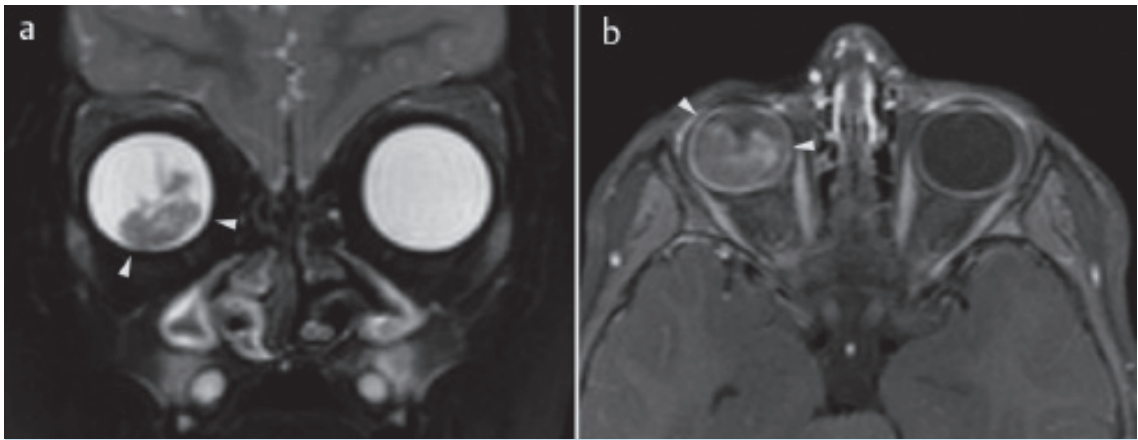

Abb. 2 Retinoblastom bei einem 3-jährigen Jungen. Das koronare T2-gewichtete Bild (a) zeigt eine große intrabulbär gelegene Raumforderung mit moderater, inhomogener KM-Aufnahme in dem transversalen fettgesättigten T1-gewichteten Bild nach KM-Gabe (b).

tum bedroht sein. Zu den häufigsten benignen Er-krankungen zählen Dermoidzysten und das kapilläre Hämangiom (Sterker I, Frerich B. Klin Monbl Augenheilkd 2006; 223: 59-67). Das Rhabdomyosarkom stellt den häufigsten malignen Tumor dar (Castillo BV, Jr., Kaufman L. Pediatr Clin North Am 2003; 50: 149-172). Die Einteilung der Orbita in anatomisch-funktionelle Kompartimente erleichtert die Differenzialdiagnostik pathologischer Veränderungen, da sich Tumoren häufig einzelnen Kompartimenten zuordnen lassen und diese Grenzen erst relativ spät durchbrechen.

Augenveränderungen im Kindesalter sind bisher selten eine Indikation für MRT-Untersuchungen, da der weitaus größte Teil der Erkrankungen durch klinische Untersuchung und Sonografie ausreichend abgeklärt werden kann. So sind die häufigsten MRT-Indikationen einerseits Erkrankungen des Retrobulbärraums und andererseits bei diffusen Erkrankungen die exakte Darstellung der Ausdehnungslokalisation.

\section{Der Bulbus}

$\nabla$

Das Retinoblastom ( 0 Abb. 1, 2) ist der häufigste maligne Tumor der Retina. Bei $90 \%$ der Kinder tritt es vor dem 5. Lebensjahr auf und ist in der Regel dorsal des Bulbusäquators gelegen. Ein bilateraler Tumor ist möglich, ebenso ein trilokuläres Auftreten mit tumoröser Beteiligung der Glandula pinealis. Das trilokuläre Retino-

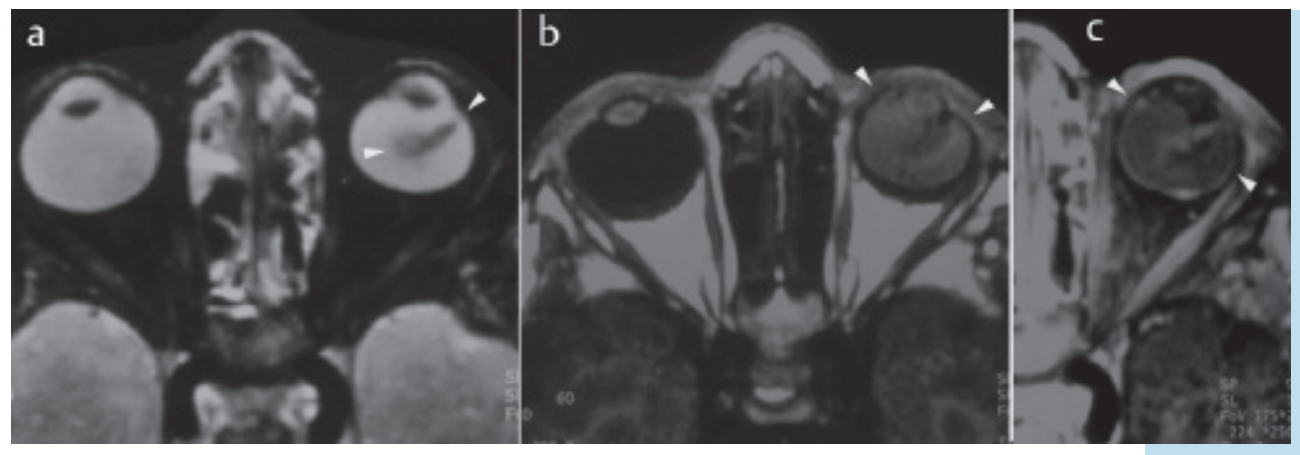

Abb. 3 Morbus Coats bei einem 10-jährigen Jungen. Die subretinalen Exudate weisen in T2-(a) und T1-gewichteten Aufnahmen (b) eine Signalanhebung auf. Diese Signalanhebungen können auch inhomogen sein, z. B. bei Vorliegen von Blutungen oder Fibrosierungen. Aufgrund der abnormen Gefäße kann in der Retina ein geringes bis moderates KM-Enhancement nachweisbar sein (c). (Figures from Müller-Forell W. Imaging of Orbital and Visual Pathway Pathology. Berlin und Heidelberg: Springer-Verlag $\mathrm{GmbH}, 2002)$. 


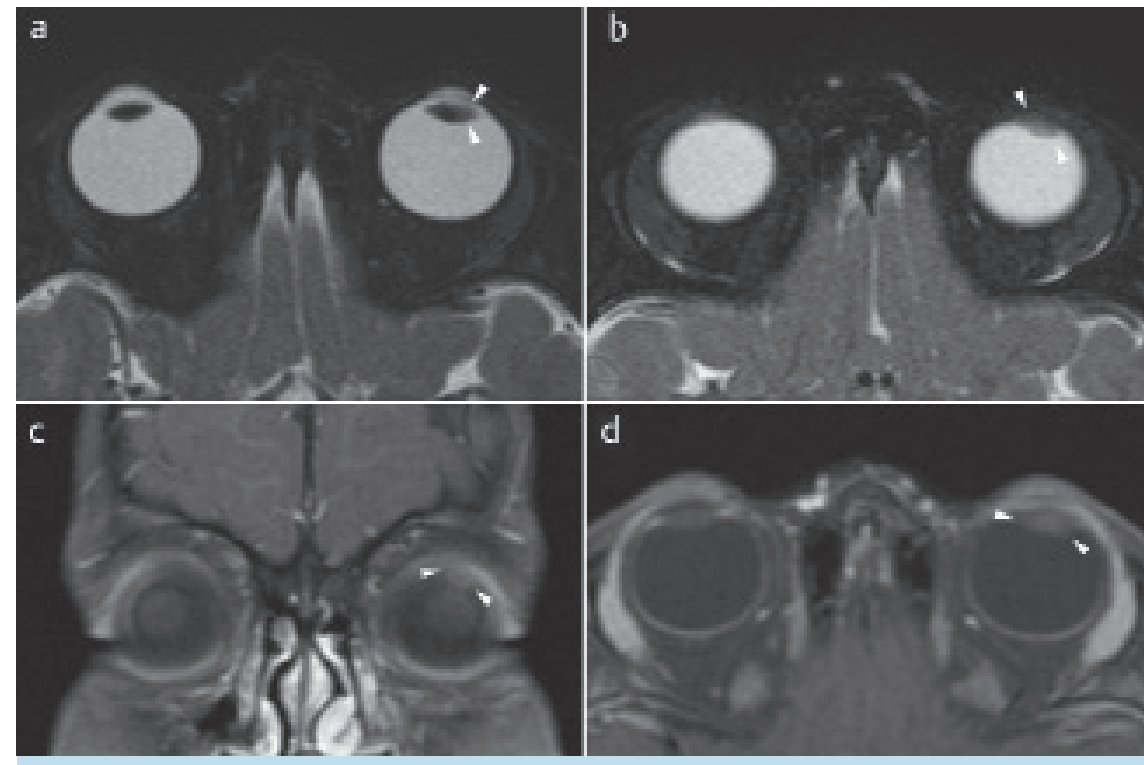

Abb. 4 Medulloepitheliom bei einem 3-jährigen Mädchen. In den axialen T2-gewichteten Aufnahmen (a, b) zeigt sich eine hypointense Raumforderung unmittelbar an den kraniolateralen Rand der Linse angrenzend. Diese weist in den T1-gewichteten Aufnahmen (c, d mit Fettsättigung) eine kräftige KM-Aufnahme auf.
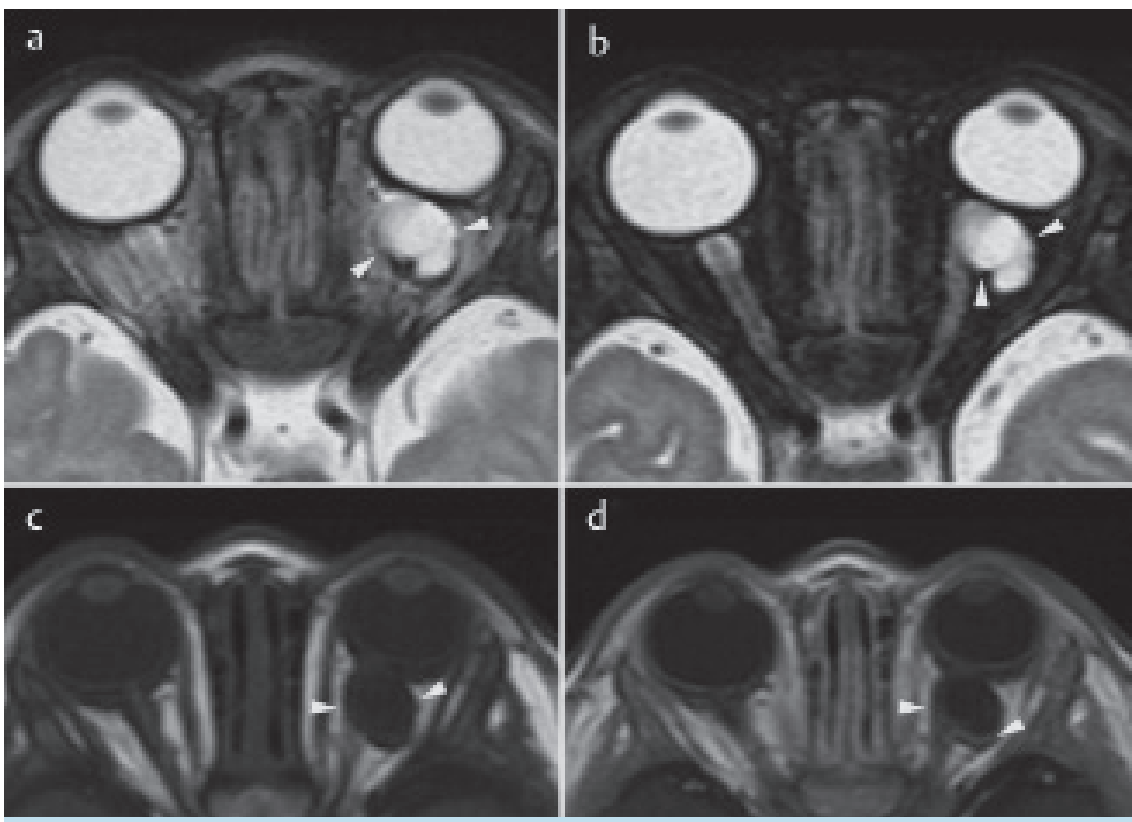

Abb. 6 Kolobom bei einem 5 Monate alten Mädchen. Nachweis einer T2-hyperintensen (a, b mit Fettsättigung) und T1-hypointensen (c nativ, $\mathbf{d}$ nach KM-Gabe) retrobulbären Raumforderung, die den N. opticus umgibt und ein diskretes Enhancement im Randbereich aufweist. Es resultiert ein im Seitenvergleich kleinerer ipsilateraler Bulbus (Mikrophthalmus).

blastom weist dabei auf die entwicklungsgeschichtliche Verwandtschaft der Pinealisdrüse mit dem Auge hin („drittes Auge“). Durch intratumorale Melaninanteile stellt sich die Raumforderung in der MRT meist T2w hypointens und in den T1w Aufnahmen hyperintens bis intermediär dar, wodurch die Abgrenzung zum Glaskörper mitunter schwierig sein kann. Es können Verkalkungen vorliegen (CT-Indikation). Zudem weist der Tumor ein deutliches KM-Enhancement auf, im Gegensatz zur wichtigsten und manchmal sehr schwierigen Differenzialdiagnose: dem Morbus Coats ( $\triangle$ Abb. 3 ). Dieser ist durch abnorme teleangiektatische retinale Gefäße gekennzeichnet, wodurch es zu einer zunehmenden Lipidablagerung mit möglicher Retinaablösung kommt. 90\% der Fälle sind unilateral und betreffen zumeist Knaben im Alter von 10 Jahren (Kandpal H, Vashisht S, Sharma R et al. Indian J Ophthalmol 2006; 54: 227-236). Die Lipidablagerungen stellen sich in beiden Wichtungen hyperintens dar und die abgehobene Retina kann aufgrund der ab-

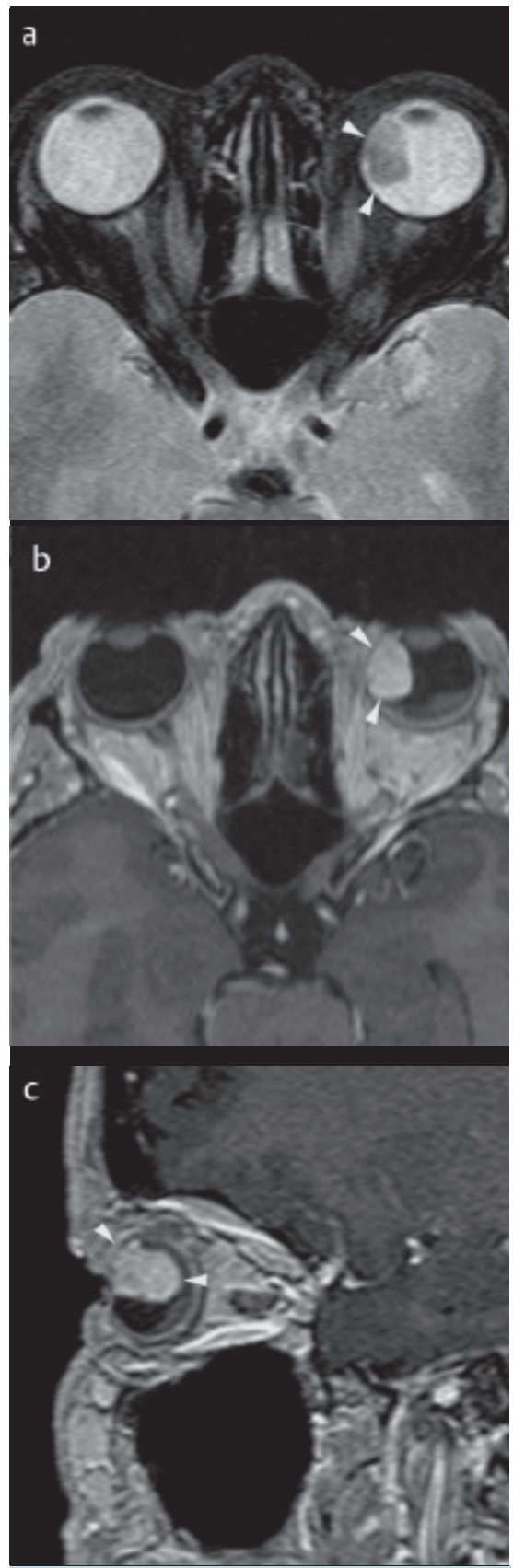

Abb. 5 Uveamelanom bei einem 6-jährigen Mädchen. Medialseitig im linken Bulbus ist eine $\mathrm{T} 2 \mathrm{w}$ hypointense Raumforderung (a) nachweisbar, welche in den kontrastverstärkten T1-gewichteten Aufnahmen (b axial, c sagittal) ein deutliches Enhancement aufweist.

normalen retinalen Gefäße ein diskretes Enhancement aufweisen. Eine weitere Differenzialdiagnose zum Retinoblastom stellt der persistierende hyperplastische primäre Glaskörper (PHPV) dar, welcher jedoch nur selten eine Indikation zur Schnittbildgebung ist.

Seltene intraokuläre Tumoren sind das Medulloepitheliom ( $\bigcirc$ Abb. 4), welches im Ciliarkörper gelegen ist, und noch sel- 

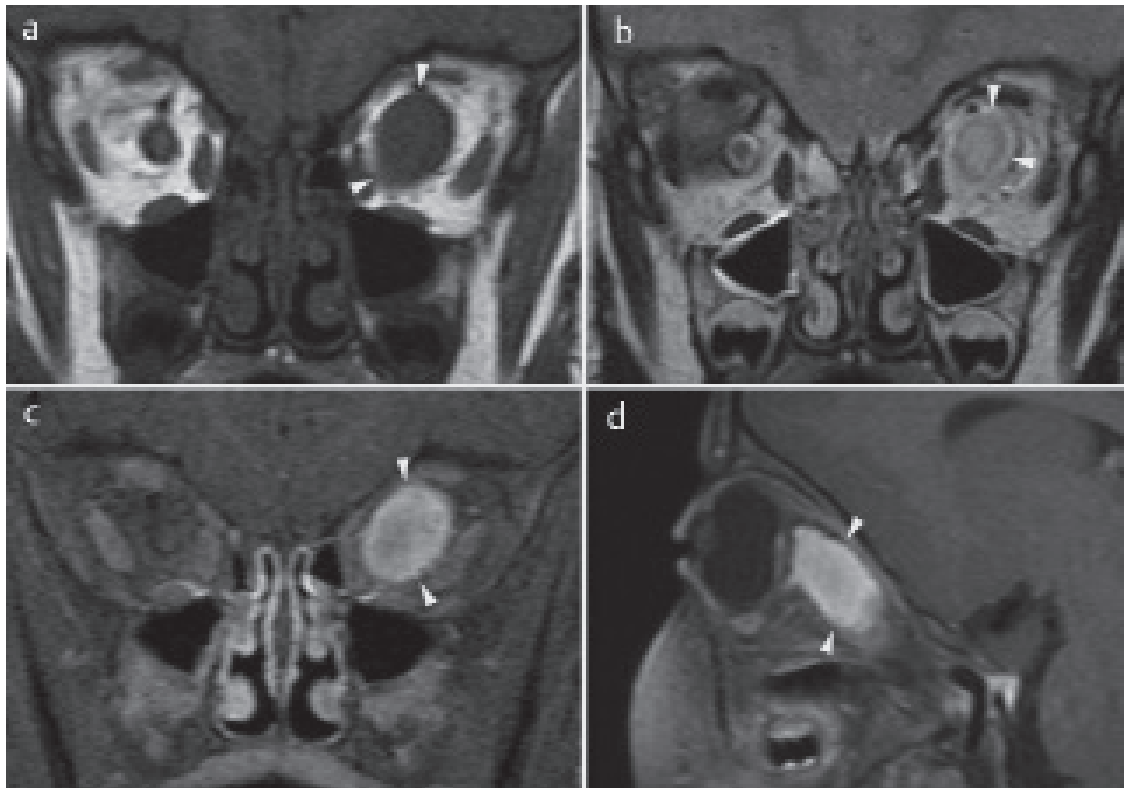

d

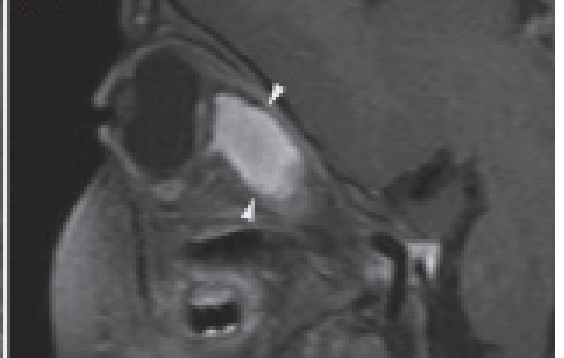

Abb. 7 Optikusgliom bei einem 31 Monate alten Mädchen. In den koronaren Aufnahmen ist eine T1hypointense (a) und T2-hyperintense (b) retrobulbäre Raumforderung darstellbar. Der N. opticus ist nicht sicher abgrenzbar und erscheint fusiform aufgetrieben. Es lässt sich eine kräftige KM-Aufnahme (c koronar, d sagittal) in den fettgesättigten T1-gewichteten Aufnahmen nachweisen.
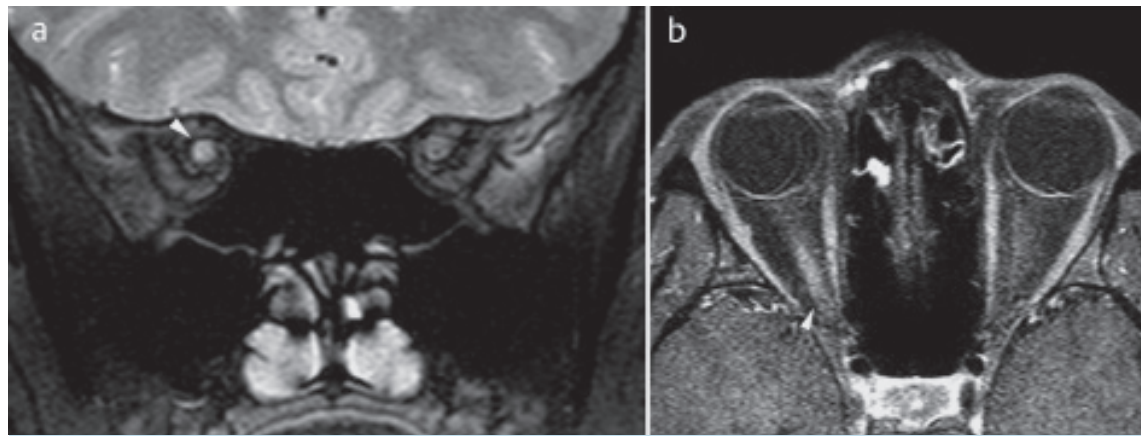

Abb. 8 Optikusneuritis bei einem 15-jährigen Mädchen. Im fettgesättigten koronaren T2-gewichteten Bild (a) und axialen T1-gewichteten Bild nach KM-Gabe (b) ist eine seitendifferente Signalanhebung im N. opticus nachweisbar. Diese ist häufig nur sehr diskret nachweisbar.
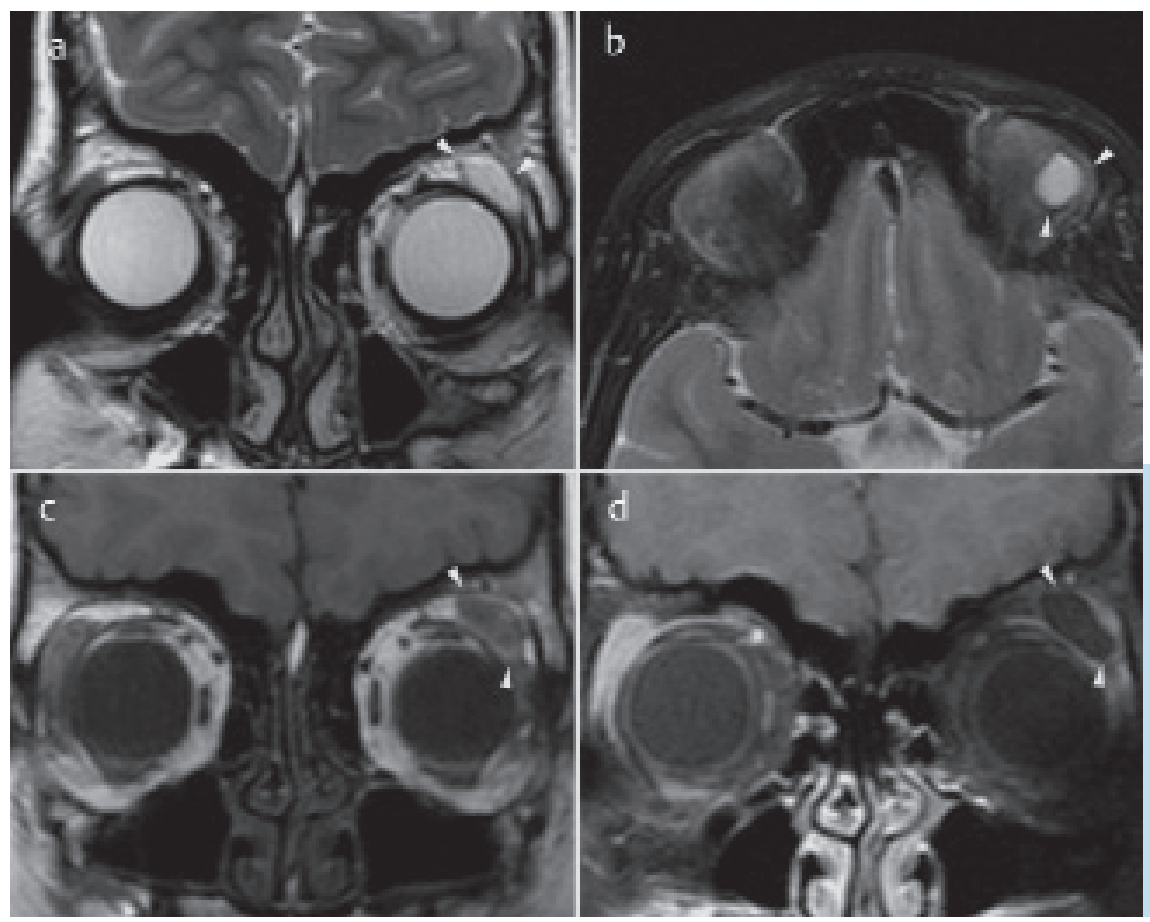

tener das Uveamelanom ( 0 Abb. 5), welches ebenso im vorderen Augenabschnitt lokalisiert ist und in Analogie zu anderen Melanomen eine Signalminderung in den T2w Bildern aufweist.

\section{Der Nervus opticus \\ $\nabla$}

Die häufigste angeborene Fehlbildung im Bereich des Sehnervs ist das Kolobom des Sehnervs ( $\bullet$ Abb. 6 ) mit auffälliger ektatischer Erweiterung der Sehnervenhüllen. Hingegen verlangen begleitende Fehlbildungen und Fehllage des N. opticus nach einer hochauflösenden MR-Bildgebung. Das im Kindesalter im Rahmen einer Neurofibromatose auftretende Opticusgliom ( $\odot$ Abb. 7) überwiegt zahlenmäßig alle Tumoren der Orbitaregion. Es findet sich eine meist deutlich KM aufnehmende Auftreibung des Sehnervs, die auch durch das Foramen n. optici hindurch bis zum Chiasma nachweisbar sein kann. Opticusgliome können prinzipiell auch isoliert außerhalb der Neurofibromatose vorkommen. Optikusscheidenmeningeome und Metastasen treten gewöhnlich erst im Erwachsenenalter auf.

Ein bildmorphologisches Korrelat der Neuritis n. optici ( $\mathbf{A}$ Abb. 8), die im Rahmen einer multiplen Sklerose zunehmend häufiger auch im Kindesalter gesehen wird, ist die ödematöse Verschwellung des Sehnervs, der sich dann gegen den umgebenden Liquor nicht mehr signalarm abgrenzen lässt, sowie die KM-Aufnahme im Nervus opticus selber.

\section{Zystische Veränderungen $\checkmark$}

Dermoide ( $\bullet$ Abb. 9 ) und epidermale Zysten ( $\odot$ Abb. 10) sind typische dysembryonale Fehlbildungen der Gesichts- und Kalottenregion und zumeist am lateralen Augenwinkel lokalisiert (Castillo M, Mukherji SK, Wagle NS. Neuroimaging Clin N Am 2000; 10: 95-116). Während sich epider-

Abb. 9 Dermoid bei einem 12-jährigen Mädchen. Das koronare (a) und fettgesättigte axiale (b) T2gewichtete Bild zeigen eine glatt begrenzte Raumforderung in Nachbarschaft zur Sutura frontozygomatica und der Tränendrüse, die zum Corpus vitreum ähnliche Signalwerte aufweist. In der nativen T1-gewichteten Aufnahme (c) ist eine geringe Signalanhebung erkennbar. Nach KM-Gabe sind in der fettgesättigten Messung (d) ein geringes Rand-Enhancement und eine zentrale Signalminderung nachweisbar. 


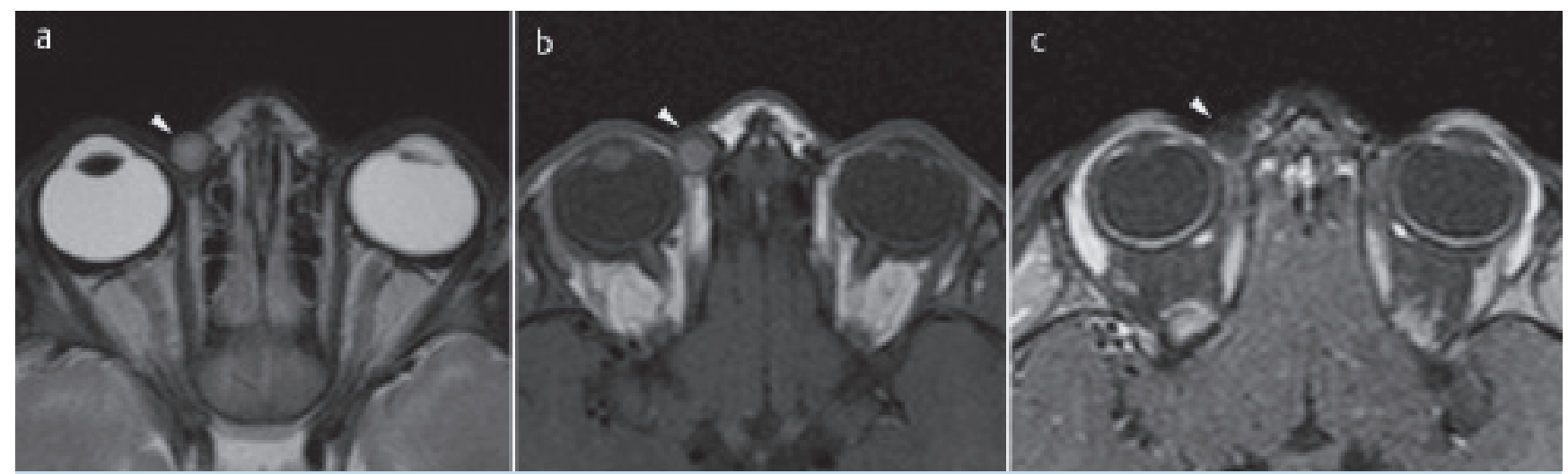

Abb. 10 Epidermale Zyste bei einem 2-jährigen Mädchen. In der axialen T2-gewichteten (a) und nativen T1-gewichteten Aufnahme (b) ist eine hyperintense, glatt begrenzte Raumforderung am inneren Augenwinkel nachweisbar. Nach KM-Gabe (c) ist ein geringes Rand-Enhancement erkennbar.

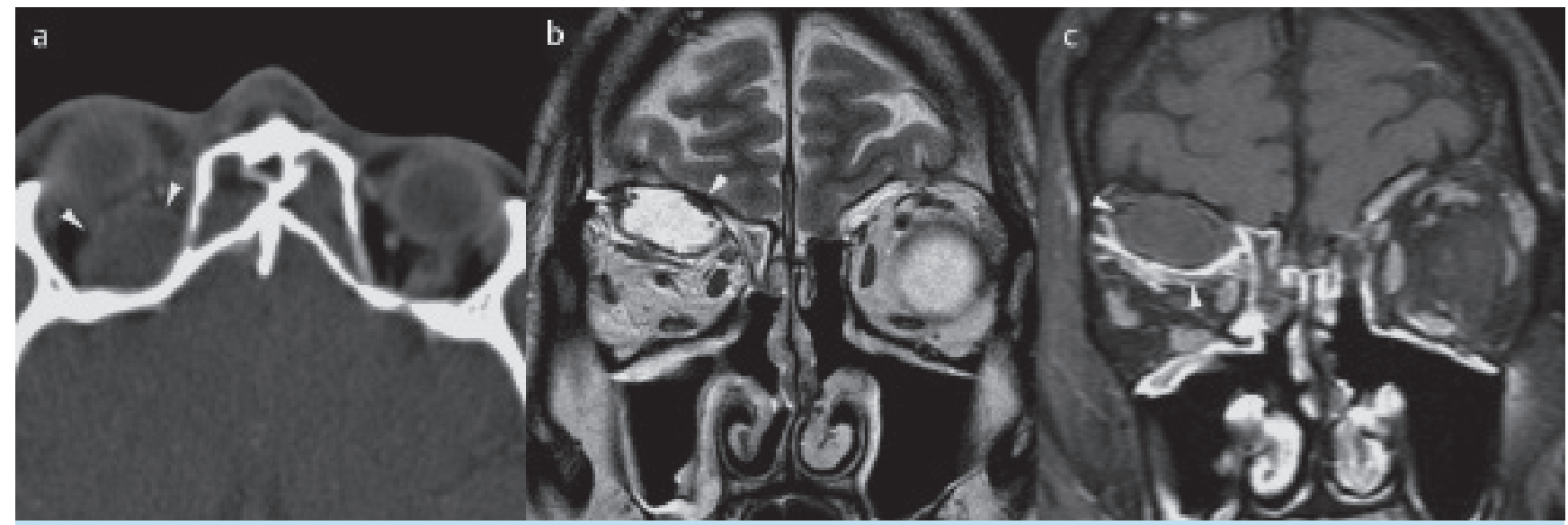

Abb. 11 Orbitale Mukozele bei einem 13-jährigen Jungen. Die axiale kontrastverstärkte CT-Aufnahme (a) zeigt eine hypodense retrobulbäre Raumforderung mit geringem Rand-Enhancement. Auf dem koronaren T2-gewichteten Bild (b) ist die Raumforderung zentral hyperintens mit hypointensem Randsaum. Korrespondierend zur CT-Untersuchung sind in der T1-gewichteten Aufnahme (c) ein Rand-Enhancement sowie eine geringe inhomogene zentrale Signalanhebung nachweisbar durch die purulenten Bestandteile.
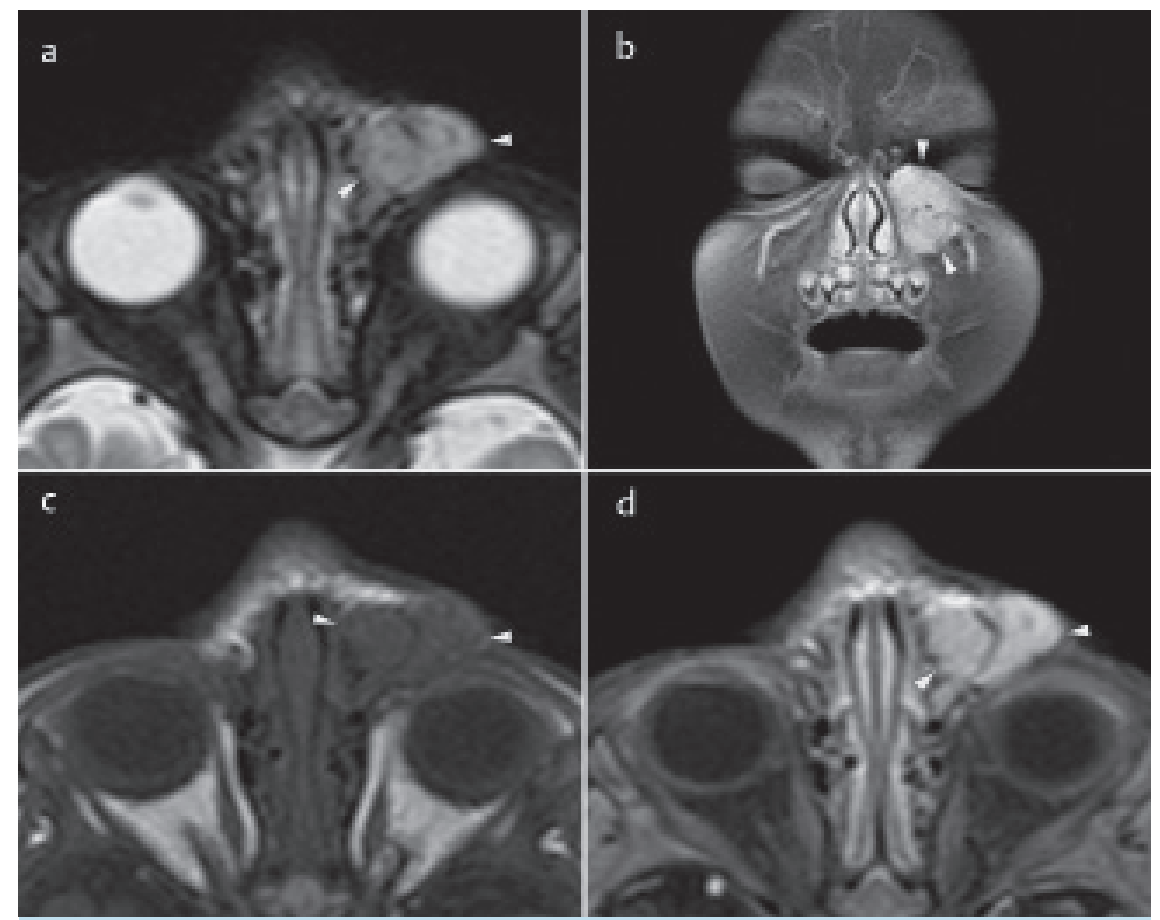

Abb. 12 Kapilläres Hämangiom bei einem 4 Monate alten Jungen. Am inneren Augenwinkel links ist eine T2-hyperintense (a) und T1-nativ hypointense (c) Raumforderung erkennbar, wodurch eine Verdrängung des linken Bulbus resultiert. Diese weist in den fettgesättigten T1-gewichteten Aufnahmen (b koronar, $\mathbf{d}$ axial) eine deutliche KM-Aufnahme auf. male Zyste als rein zystische Läsionen darstellen und somit ein erhöhtes Signal auf diffusionsgewichteten Bildern zeigen, können Dermoide auch andere Gewebebestandteile, wie Talgdrüsen oder Fett, aufweisen.

Mukozelen ( $\triangle$ Abb. 11) in der Orbita können durch embryologisch versprengte Schleimhaut vorkommen, aber auch durch Sinus-Mukozelen entstehen, welche in die Orbita einbrechen. Orbitale Mukozelen sind insgesamt selten, da die Entwicklung der Stirnhöhlen meist erst im frühen Erwachsenenalter abgeschlossen ist.

\section{Vaskuläre Veränderungen}

$\nabla$

Das kapilläre Hämangiom ( 0 Abb. 12) ist der häufigste vaskuläre Tumor und meist schon kongenital oder kurz nach der Geburt vorhanden (Plesner-Rasmussen $\mathrm{HJ}$, Marushak D, Goldschmidt E. Acta Ophthalmol (Copenh) 1983; 61: 645-654). Sie gehen, wie andere av-Angiodysplasien, 

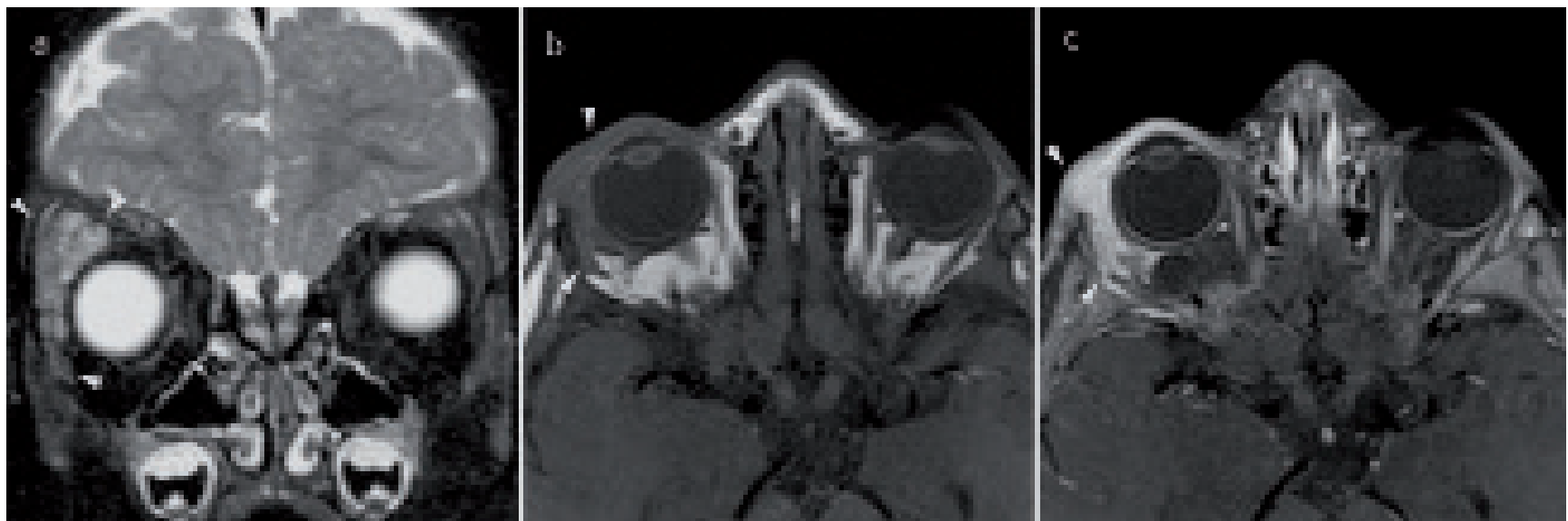

Abb. 13 Odontogenes Myxom bei einem 17 Monate alten Jungen. Auf der koronaren T2-gewichteten Aufnahme (a) ist eine inhomogene hyperintense Raumforderung zwischen Bulbus und Os zygomaticus erkennbar. Diese ist in der nativen T1-gewichteten Aufnahme (b) hypointens, mit starker Signalanhebung in der konstrastverstärkten fettgesättigten T1-gewichteten Aufnahme (c). Eine Infiltration des Bulbus ist nicht nachweisbar.

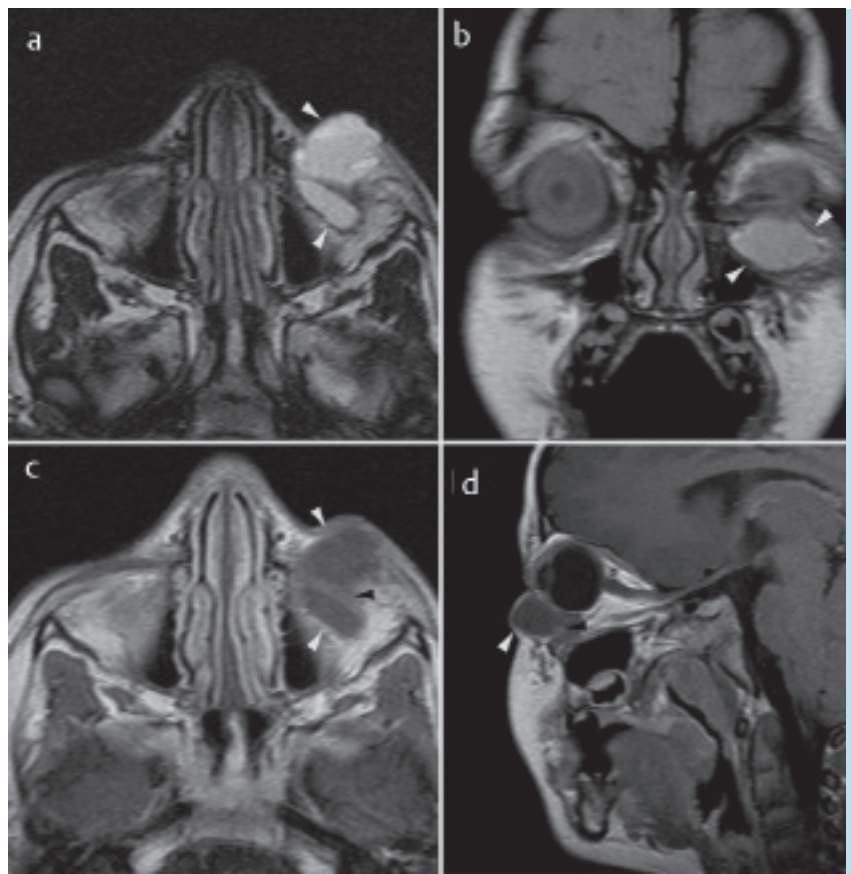

Abb. 14 Lymphangiom bei einem 32 Monate alten Mädchen. Die in T2- (a) und PD-Wichtung (b) hyperintense infraorbital gelegene und septierte Raumforderung führt zu einer geringen Dorsalverlagerung des Bulbus. Nach KM-Gabe zeigt sich eine diskrete Signalanhebung der Septen und des Randbereichs in den T1-gewichteten Aufnahmen (c, d). überwiegend vom Extrakonalraum aus und weisen eine kräftige KM-Aufnahme auf, bei heterogener Signalgebung in der Nativdiagnostik. Ausschließlich bildgebend ist eine Differenzierung zwischen den verschiedenen Angiodysplasien, aber auch zu anderen hypervaskularisierten Tumoren wie dem odontogenen Myxom ( Abb. 13) nicht möglich.

Im Gegensatz dazu weist das Lymphangiom ( Abb. 14) nur ein geringes (bis kein) Enhancement im Randbereich und in den Septen auf mit dazwischen gelegenen zystischen Strukturen.

\section{Neoplasien}

$\nabla$

Das Rhabdomyosarkom ( $\bullet$ Abb. 15) ist die häufigste orbitale Neoplasie im Kindesalter (Altersgipfel: 8-9 Jahre) und weist
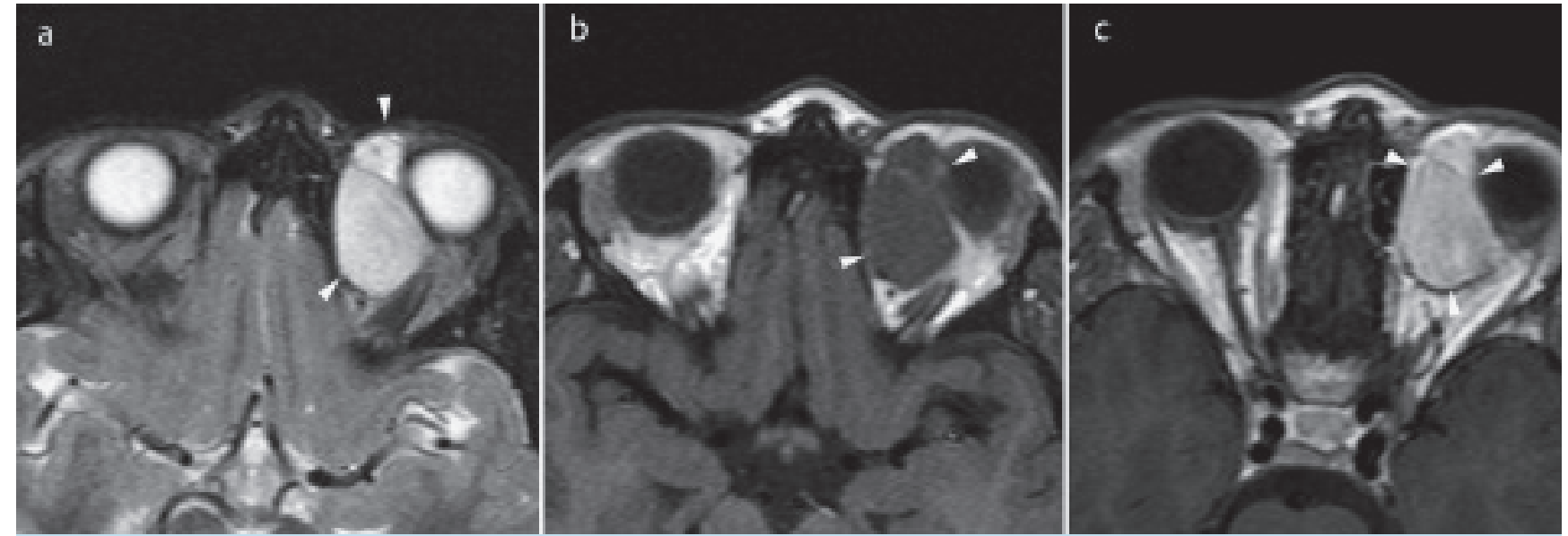

Abb. 15 Rhabdomyosarkom bei einem 8-jährigen Jungen. Die ausgedehnte T2-hyperintense (a) und T1-hypointense (b) Raumforderung mit deutlichem Enhancement (c) liegt der Lamina paryracea unmittelbar an und führt zu einer Verdrängung des Bulbus. Vom retrobulbären Fettgewebe und dem Bulbus selbst ist sie jedoch glatt abgrenzbar. 


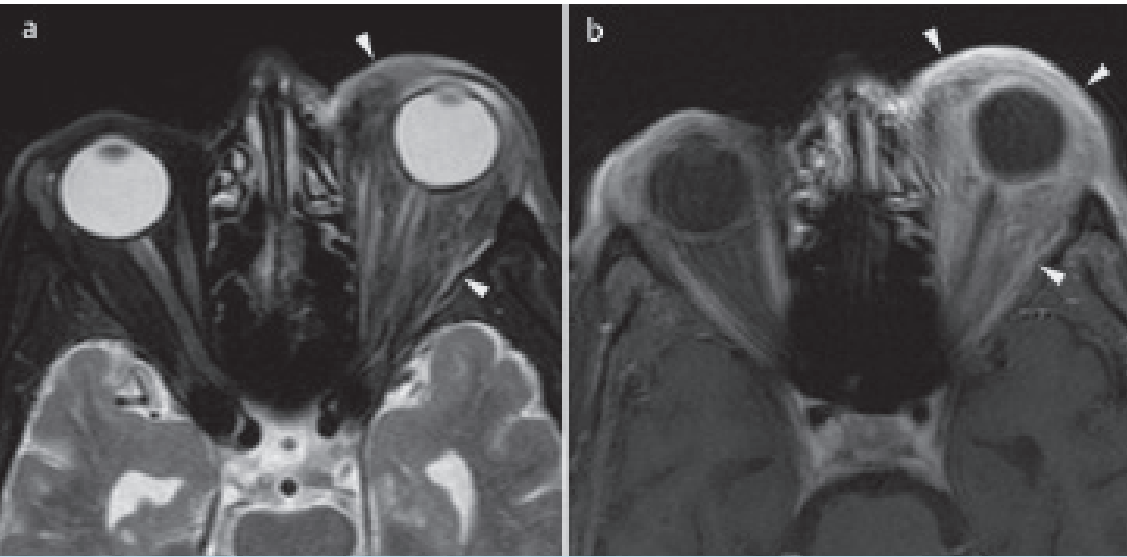

Abb. 16 Orbitaphlegmone bei einem 12-jährigen Jungen. Auf den axialen fettgesättigten T2-gewichteten (a) und kontrastverstärkten T1-gewichteten (b) Aufnahmen ist eine deutliche inhomogene Signalanhebung innerhalb der deutlichen Lidschwellung nachweisbar. Das retrobulbäre Fettgewebe ist mitbeteiligt, eine Abszessformation ist jedoch nicht zu erkennen.
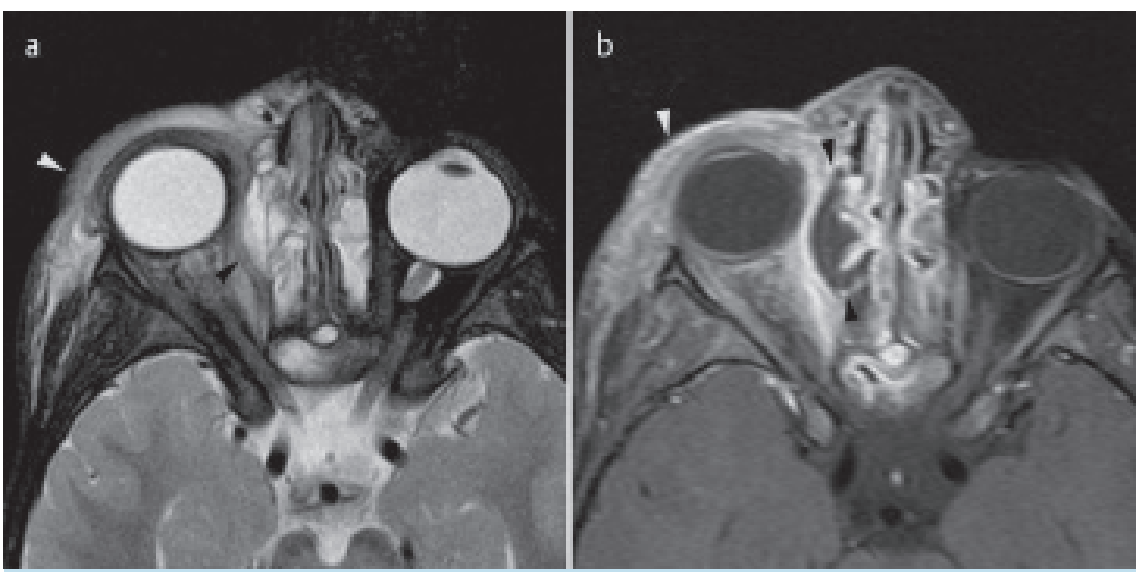

Abb. 17 Subperiostaler Abszess bei einem 13-jährigen Mädchen. Auf der axialen fettgesättigten T2gewichteten (a) und der kontrastverstärkten T1-gewichteten (b) Aufnahme ist eine präseptale Zellulitis (weißer Pfeil) erkennbar, die bis in das retrobulbäre Fettgewebe reicht. Zusätzlich lässt sich jedoch ein subperiostaler Abszess der Lamina paryracea (schwarzer Pfeil) nachweisen. Ursächlich ist a.e. die angrenzende floride Sinusitis.
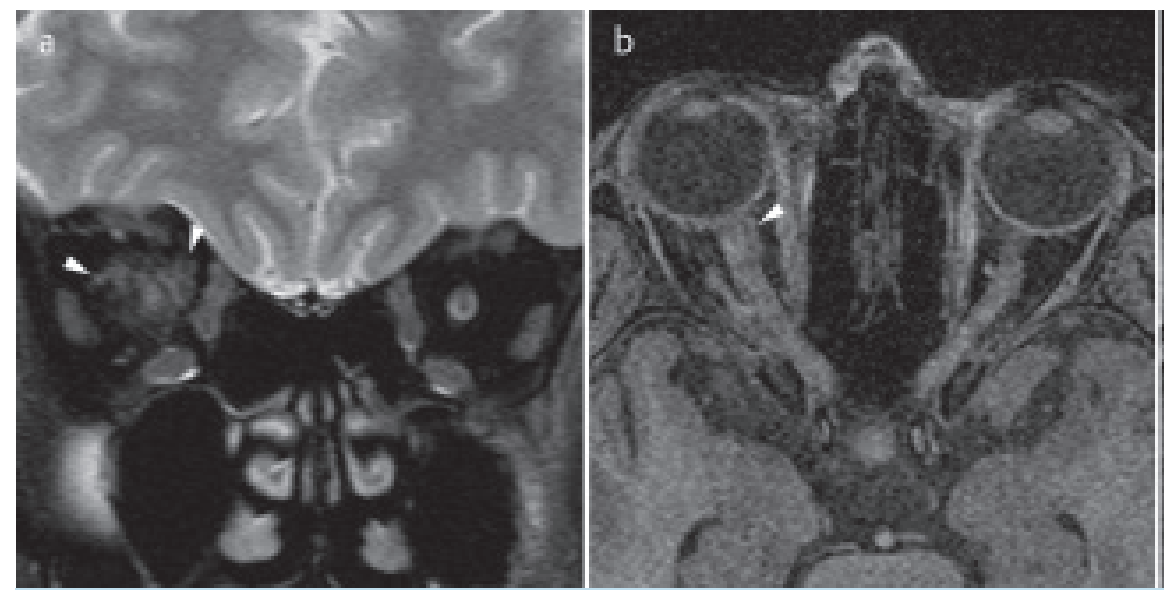

häufig eine rasch progrediente Proptosis auf (Barnes PD, Robson CD, Robertson RL et al. Neuroimaging Clin N Am 1996; 6: 179-198). Sie geht im Regelfall von mesenchymalen Zellen des Retrobulbärraums aus, kann den Intra- und Extrakonalraum betreffen, zu einer Infiltration der angrenzenden Knochenstrukturen führen und nach intrakraniell infiltrieren.

Lymphome können unilateral oder bilateral auftreten, alle Kompartimente betreffen und sowohl als Primärbefund als auch im Rahmen einer systemischen Erkrankungen auftreten. Bei insgesamt sehr geringer Inzidenz ist am häufigsten die Beteiligung der Tränendrüse mit resultierender Lidschwellung beschrieben (Valvassori GE, Sabnis SS, Mafee RF et al. Radiol Clin North Am 1999; 37: 135-150).

\section{Entzündliche Veränderungen $\nabla$}

Entzündliche Erkrankungen bei Kindern sind ganz überwiegend von den Nasennebenhöhlen permeativ fortgeleitet. Zu unterscheiden sind vor allem die klinischen Stadien der präseptalen und orbitalen Entzündungen ( $\triangle$ Abb. 16), die keiner operativen Therapie bedürfen, von abszedierenden Veränderungen ( $\bullet$ Abb. 17), die sich ganz überwiegend subperiostal manifestieren.

Eine weitere Form stellt die idiopathische orbitale Entzündung ( $\bullet$ Abb. 18) dar, welche mit einer großen Anzahl von unterschiedlichen Entitäten assoziiert ist, die alle eine entzündliche Infiltration des intraorbitalen Fettgewebes unklarer Ätiolo-

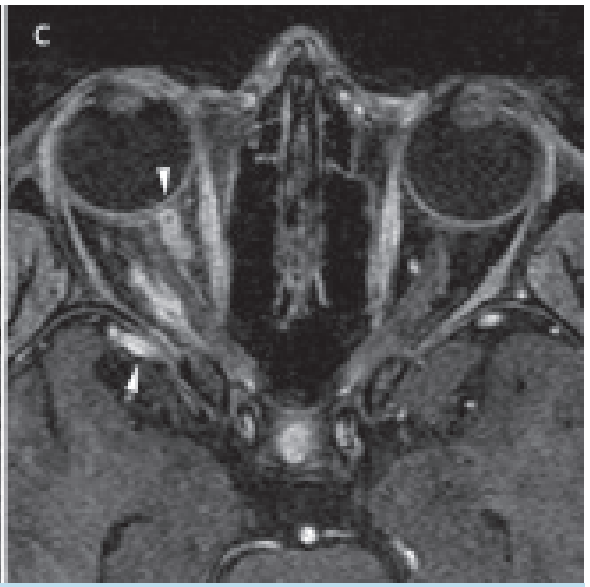

Abb. 18 Idiopathischer Orbitatumor bei einem 12-jährigen Jungen. In der koronaren T2-gewichteten Aufnahme (a) ist eine diffuse ödematöse Infiltration des retrobulbären Fettgewebes erkennbar, die nur unscharf vom N. opticus abgrenzbar ist. Nativ T1-hypointens (b, fettgesättigt) ist typischerweise eine Signalanhebung nach KM-Gabe nachweisbar (c, fettgesättigt). 

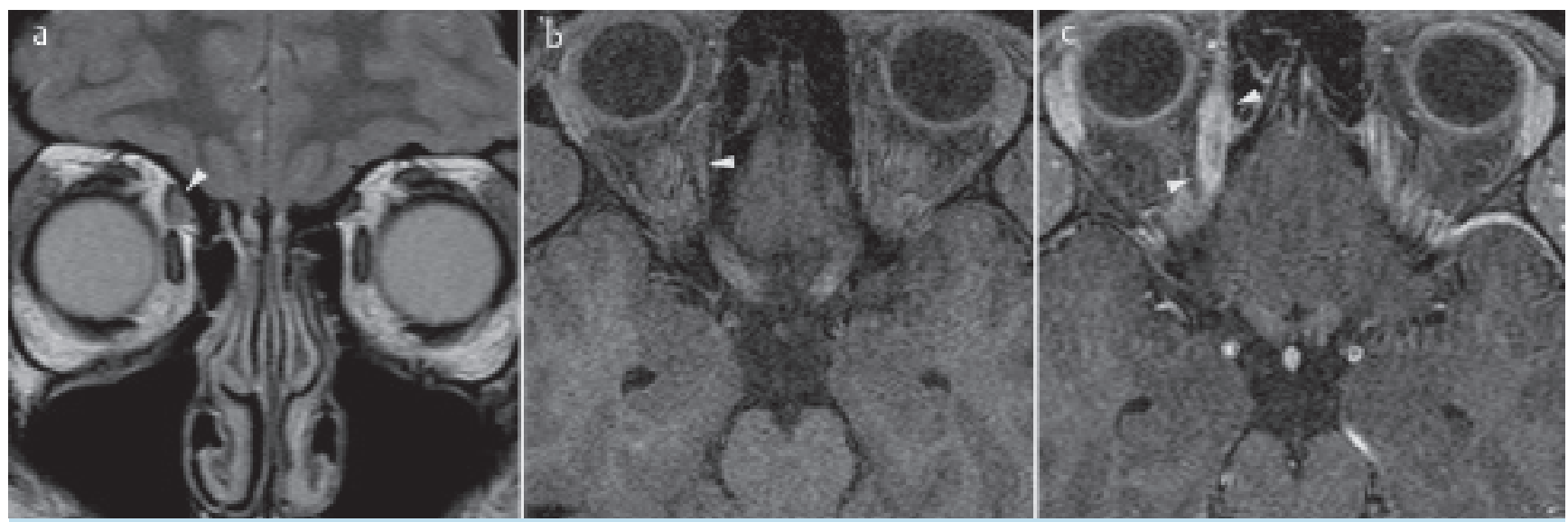

Abb. 19 Myositis bei einem 16-jährigen Mädchen. In der koronaren PD-gewichteten Aufnahme (a) ist ein seitenasymmetrisch aufgetriebener und signalangehobener M. obliquus superior rechts nachweisbar. Isointens zu den übrigen Muskeln in der nativen T1-Wichtung (b) ist ein fokal begrenztes Enhancement nach KM-Gabe (c) nachweisbar.

gie gemein haben. Eine dieser Entitäten ist die Myositis (@Abb. 19) eines Augenmuskels - dies ist zwar selten im Kindes- und Jugendalter, kann aber dann wegen der isolierten Zuordnung des Befunds zu einem Muskel sicher differenziert werden.

\section{Zusammenfassung \\ $\nabla$}

Aufgrund der Seltenheit von Orbitaerkrankungen im Kindesalter ist deren Kenntnis für eine effiziente Diagnostik und erfolgreiche Therapie sowohl für den Erhalt des Visus als auch des Lebens der Kinder von entscheidender Bedeutung. Vor allem Ultraschall und MRT können in
Kombination mit der klinischen Untersuchung in den meisten Fällen die korrekte Diagnose stellen. Die Aufgabe der MRT ist vor allem die Beurteilung der exakten Ausdehnung von Tumoren und Entzündungen.

Reiss-Zimmermann M, Sorge I, Sterker I, Hirsch W, Leipzig

\section{Erratum}

„Reiss-Zimmermann M, Sorge I, Sterkerl, Hirsch W. MRT-Bildgebung von Orbitaerkrankungen im Kindesalter.Fortschr Röntgenstr 2011; 183(09): 781-787“

Fig. 3a-c was unintentionally published without permission of the copyright owner and without indication of the original source. The correct source of Fig. 3a-c is as follows: Müller-Forell W. Imaging of Orbital and Visual Pathway Pathology. Berlin \& Heidelberg: Springer-Verlag GmbH, 2002.

The article was changed on May 11, 2016. 